\title{
Immunogenicity and protective potency of Norovirus GII.17 virus-like particle-based vaccine
}

\author{
Wei Chen · Tao Kang $\cdot$ Rongliang Yuan $\cdot$ Congwen Shao $\cdot$ Shenrong Jing $\mathbb{D}$
}

Received: 22 October 2019/Accepted: 10 February 2020/Published online: 22 February 2020

(C) Springer Nature B.V. 2020

\begin{abstract}
Objectives Noroviruses (NoVs) are major cause of acute viral gastroenteritis in worldwide, and the lack of a cell culture system that must be considered the virus like particles (VLPs) are used as an effective vaccine development.

Materials and methods In the present study, we investigated the expression of the major capsid protein (VP1) of the Genogroup II, genotype 17 (GII.17) NoV, using recombinant baculovirus system in insect cells, as well as a saliva binding blockade assay to detect their protective potency.

Results Our results showed that GII.17 VLPs could be successfully generated in sf9 insect cells, and electron microscopic revealed that GII.17 VLPs appeared as spherical particles with a $-35 \mathrm{~nm}$ diameter. Immunized mice with purified VLPs produced GII.17 specific sera and could efficiently block
\end{abstract}

Wei Chen and Tao Kang have equally contribution to the paper.

W. Chen $\cdot$ T. Kang $\cdot$ S. Jing $(\bowtie)$

Medical School, Kunming University of Science and

Technology, No. 727, Southern Jingming Road,

Chenggong District, Kunming 650500,

Yunnan, China

e-mail: jingshenrong@kmust.edu.cn

R. Yuan - C. Shao

Wuxi Huapai Biotechnology Co., Ltd, Wuxi,

Jiangsu, China
GII.17 VLPs binding to the saliva histo-blood group antigens (HBGAs).

Conclusions Together, these results suggested that GII.17 VLPs represent a promising vaccine candidate against NoV GII.17 infection and strongly support further preclinical and clinical studies.

Keywords Norovirus GII.17 · Saliva histo-blood group antigens (HBGAs) · sf9 cells · Vaccine · Virus like particles (VLPs)

\section{Introduction}

Human noroviruses (NoVs) infections are considered a significant public health problem, which cause more than $50 \%$ of non-bacterial gastroenteritis characterized by fever, vomiting and frequent episodes of diarrhea, especially in adults and children (Ahmed et al. 2014; Kaplan et al. 1982). NoVs are divided into 5 genogroups designated GI through to $\mathrm{GV}$ and subdivided into 9 and 22 genotypes, which were first linked to human disease in 1972 (Kroneman et al. 2013; Zheng et al. 2006). From the mid-1990s, Genogroup II, genotype 4 (GII.4) NoV have predominantly occored as outbreaks, which emergence with new variants every two or three years (Ramani et al. 2014). However, since the emergence of the novel Genogroup II, genotype 17 (GII.17) Kawasaki_2014 
strain, a new pandemic strain has become predominant in the world. The novel GII.17 not only caused NoVs outbreaks but also spread sporadically with cases reported in China (Shanghai, Taiwan) and USA during the winter of 2014_2015 (Chen et al. 2015; Lee et al. 2015; Parra and Green 2015). From 2015, NoV GII.17 continued to spread to many countries in South America, Europe and Asia (Andrade et al. 2017; Huang et al. 2017; Khamrin et al. 2016). These epidemiological surveys indicated that NoV GII.17 is becoming an important etiological agent of gastroenteritis.

NoVs belongs to the family of Caliciviridae and are nonenveloped, single-stranded, positive-sense ribonucleic acid (RNA) viruses. The genomes are 7.4-7.7 kb in size and are typically organized into three open reading frames (ORFs). The ORF1 encodes nonstructural proteins, such as the RNA dependent RNA polymerase (RdRp), while the structural proteins, such as the major and minor capsid protein (VP1 and VP2), are encoded by ORF2 and ORF3, respectively (Glass et al. 2009). VP1 is a $60 \mathrm{KDa}$ protein that can be divided into a shell domain ( $\mathrm{S}$ domain) and a protruding domain (P domain). The $\mathrm{S}$ domain forms a scaffold surrounding the viral RNA and is responsible for the shell structure of the capsid, whereas the $\mathrm{P}$ domain contains neutralizing epitopes and binds to the histo-blood group antigens (HBGAs) receptors, responsible for the genetic variability, builds the viral spikes and facilitates cell attachment (Debbink et al. 2012; Prasad et al. 1999; Tan and Jiang 2005). VP2, a minor capsid protein, exhibit multiple functions, which are observed to stabilize and promote expression of VP1 (Lin et al. 2014).

The most recent outbreak of NoV GII.17 strain has been sweeping all over the world; however, there are no available vaccines or specific treatments. Due to the lack of a permissive cell culture system or available animal models, the main capsid protein VP1 that forms the virus like particles (VLPs) has been assembled and used as a NoVs vaccine candidates in preclinical and clinical studies (Lucero et al. 2017). VLPs have shown high immunogenicity, safety and promising results as human enteroviruses vaccines, such as enterovirus 71 (EV71), coxsackievirus A16 (CA16), and enterovirus D68 (EV-D68) (Dai et al. 2018; Zhang et al. 2015, 2016).

In the present study, we constructed the GII.17 VP1, which was expressed using the recombinant baculovirus expression system in sf9 cells, which lead to the formation of VLPs that are morphologically and antigenically similar to the true virions. Our results showed that GII.17 VLPs could be readily produced in the baculovirus/insect cell system and these VLPs could induce potent neutralizing antibody responses and provided effective protection.

\section{Materials and methods}

\section{Construction of baculovirus expression vectors}

The full-length capsid protein VP1 coding sequence (Genbank number, KT992785) was optimized based on insect cell expression and synthesized by Sangon Biotech (Shanghai, China) with flanked by BamHI and $X b a 1$ sites at its $5^{\prime}$ and $3^{\prime}$ ends, respectively. The optimized gene was cloned into the pFastBac-DualEGFP vector (our laboratory construction previously), named pFastBac-Dual-EGFP-VP1 (GII.17). Subsequently, the pFASTBac-Dual-EGFP-VP1 (GII.17) vector purified plasmid DNA was transformed into DH10Bac ${ }^{\mathrm{TM}} E$. coli for transposition into the bacmid. Finally, the correct recombinant bacmid DNA was transfected into sf9 insect cells to produce recombinant baculovirus.

\section{Production and purification of NoV GII.17 VLPs}

After $96 \mathrm{~h}$ of transfection (green fluorescence reached its optimal point), the budded virus was released into the medium and collected the medium, the P1 viral stock. Amplified baculoviral was stocked until P5 viral stock and collected cells.

Cells were washed and lysed in a phosphate buffer solution (PBS) using ultrasound. The lysates were supplemented with $0.5 \mathrm{~mol} / \mathrm{L} \mathrm{NaCl}$ and $10 \%$ (W/V) PEG 8000 was mixed for $6 \mathrm{~h}$, and subsequently centrifuged at $10,000 \times g$ for $30 \mathrm{~min}$. The resultant precipitates were loaded onto a $20 \%$ sucrose cushion, and subjected to $10-50 \%$ sucrose gradients for ultracentrifugation at $40,000 \times g$ for $6 \mathrm{~h}$. After this, fractions were taken from top to the bottom and then analyzed for its contents of VLPs by SDS-PAGE. The uninfected sf9 cells were subjected to the same treatments as the negative control. The final purified proteins were dialyzed with physiological saline at 
$4{ }^{\circ} \mathrm{C}$ overnight and used for immunization experiments.

\section{Electron microscopy}

Purified GII.17 VLPs was negatively stained with $0.5 \%$ aqueous uranyl acetate, and observed by transmission electron microscopy with Tecnai G2 Spirit at $120 \mathrm{~V}$ (Thermo, USA).

\section{Mouse immunization}

Mice were purchased from Kunming Medical University (Yunnan, China). All animal studies were approved by the Institutional Animal Care and Use Committee at the Kunming University of Science and Technology.

GII.17 VLPs or the negative control antigen were adsorbed to the aluminum hydroxide adjuvant with 1:1 by vortexing to a final volume of $100 \mu \mathrm{L}$ containing $50 \mu \mathrm{g}$ of GII.17 VLPs for each injection. Groups of 6 female BALB/c mice (6-8 weeks old) were intraperitoneally (i.p.) injected with antigen mixtures at weeks 0 and 4. Blood samples were collected from each immunized mouse after the final immunization, and placed at $37{ }^{\circ} \mathrm{C}$ for $2 \mathrm{~h}$ and $4{ }^{\circ} \mathrm{C}$ for overnight.

Serum antibody measurement assay

NoV GII.17 specific antibodies in immunized mice serums were detected by indirect ELISA assays. 96-well plates were coated with $0.1 \mu \mathrm{g}(100 \mu \mathrm{L}) /$ well of GII.17 VLPs at $4{ }^{\circ} \mathrm{C}$ overnight, followed by blockade with $5 \%$ milk diluted in PBS containing $0.05 \%$ Tween-20 (PBS-T) at $37^{\circ} \mathrm{C}$ for $1 \mathrm{~h}$. Subsequently, they were incubated at $37^{\circ} \mathrm{C}$ for $2 \mathrm{~h}$ with 100 $\mu \mathrm{L} /$ well of serum samples in which individual serum samples were diluted in series in PBS-T. Finally, incubation with goat anti-mouse IgG HPR (Abcam, Ab6789, UK) at $37{ }^{\circ} \mathrm{C}$ for $1 \mathrm{~h}$. Between each step, the plates were washed three times with PBS-T. The absorbance was measured at $450 \mathrm{~nm}$ using a microplate reader (Thermo, USA).

\section{Western blot assay}

Collected mouse sera were used to determine their reactivates on VP1 protein. In brief, the VLPs proteins separated by SDS-PAGE were transferred to a PVDF membrane and detected using mouse anti-GII.17 VLPs serum at a dilution of 1:20,000 in PBS-T and then HRP conjugated goat anti-rabbit IgG polyclonal antibody was added at 1:10,000 in an enzyme buffer. The membrane was developed after a subsequent three times of wash using PBS-T with DAB.

\section{GII.17 VLPs-HBGAs binding assay in vitro}

For the in vitro VLPs-HBGA binding assay, the eighty-four saliva samples collected from blood type $\mathrm{A}, \mathrm{B}, \mathrm{AB}$ and $\mathrm{O}$ individuals were used. The protocol for the GII.17 VLPs saliva HBGAs binding assay was conducted based on previously description (Huang et al. 2003; Marionneau et al. 2005). Briefly, human saliva with known $\mathrm{ABO}$ antigens was boiled for $10 \mathrm{~min}$ and diluted at 1:1000 in PBS, added into 96-well plates $\left(100 \mu \mathrm{L} /\right.$ well) and incubated at $4{ }^{\circ} \mathrm{C}$ for overnight. After which, it was by blocked with $5 \%$ milk diluted in PBS-T at $37{ }^{\circ} \mathrm{C}$ for $1 \mathrm{~h}$. GII.17 VLP at $0.1 \mu \mathrm{g}(100 \mu \mathrm{L}) /$ well was incubated at $37{ }^{\circ} \mathrm{C}$ for $2 \mathrm{~h}$, followed by dilution of serum samples; 1:1000 PBS-T at $37{ }^{\circ} \mathrm{C}$ for $2 \mathrm{~h}$ with $100 \mu \mathrm{L} /$ well. At last, samples were incubated with goat anti-mouse IgG HPR (Abcam, Ab6789, UK) at $37^{\circ} \mathrm{C}$ for $1 \mathrm{~h}$. Between each step, the plates were washed three times with PBS-T. The absorbance was measured at $450 \mathrm{~nm}$ using a microplate reader. At the same time, we used PBS-T without sera as a negative control.

\section{GII.17 VLPs-HBGAs binding blockade assay}

The blocking activity of sera against GII.17 VLPs was determined by an in vitro VLPs-HBGAs binding blockade assay. The protocol was performed practically at the same as the in vitro VLPs-HBGA binding assay except for serum dilution and antibody. Since GII.17 VLPs exhibited the strongest affinity to blood type AB salivary HBGAs, a blood type A saliva sample was selected for the blockade assay. For all reagents, sera dilutions and reaction times were optimized, and we conducted saliva-VLPs blocking assays using the mouse sera. The 96-well microplates were added to purified VLPs containing VLPs mice serum at $37{ }^{\circ} \mathrm{C}$ for $1 \mathrm{~h}$, and diluted rabbit anti-GII.17$\mathrm{P}$ domain monoclonal antibodies (Taizhou SCIVAC Bio-Tech CO., Ltd) were added. Wells added with VLPs that did not contain VLPs in the mice serum were selected as controls. The blocking index was 
calculated in \% as (mean OD without VLP sera-mean OD with VLP sera)/mean OD without VLP sera $x$ $100 \%$ (Huo et al. 2016b).

\section{Statistical analysis of data}

Statistical analyses were performed using Graphpad Prism v5 software. Antibody titers or OD values between groups were compared by Student's twotailed t-test.

\section{Results}

Expression and characterization of NoV GII.17 VLPs in sf9 cells

In order to confirm the expression of GII.17 VP1 protein in sf9 cells, EGFP and VP1 genes were cloned together into a pFastBac-Dual vector under the control of $\mathrm{pH}$ and $\mathrm{p} 10$ promoters, respectively (Fig. 1a). We used for the recombinant baculovirus was a Bac-toBac baculovirus expression system and subsequently used it to infect insect cells. When green fluorescence reached its optimal level under the fluorescence microscope, we collected the cells. Sucrose gradient ultracentrifugation was used to purify the VP1 revealed by SDS-PAGE and the results, which corresponds to the full-length VP1 protein of $-58 \mathrm{KDa}$ (Fig. 1b). The purity and integrity of VLPs were showed by transmission electron microscopy (Fig. 1c). It should be noted that smaller, larger and full VLPs (about $35 \mathrm{~nm}$ ) were observed.

Specificity of the antibody response following immunization

To determine the immunogenicity, GII.17 VLPs was used to i.p. immunize BALB/c mice three times after four weeks. Another group of mice were injected with PBS as a control. Serum samples were collected 2 weeks after the final immunization and were subjected to ELISA analysis for antibody measurement. All sera from VLPs immunized mice exhibited high binding affinities towards GII.17 VLPs, whereas the control group did not exhibit significant reactivity (serum 1:20,000 dilution) (Fig. 2a). As showed in (Fig. 2b) Western blot analysis confirmed the

A
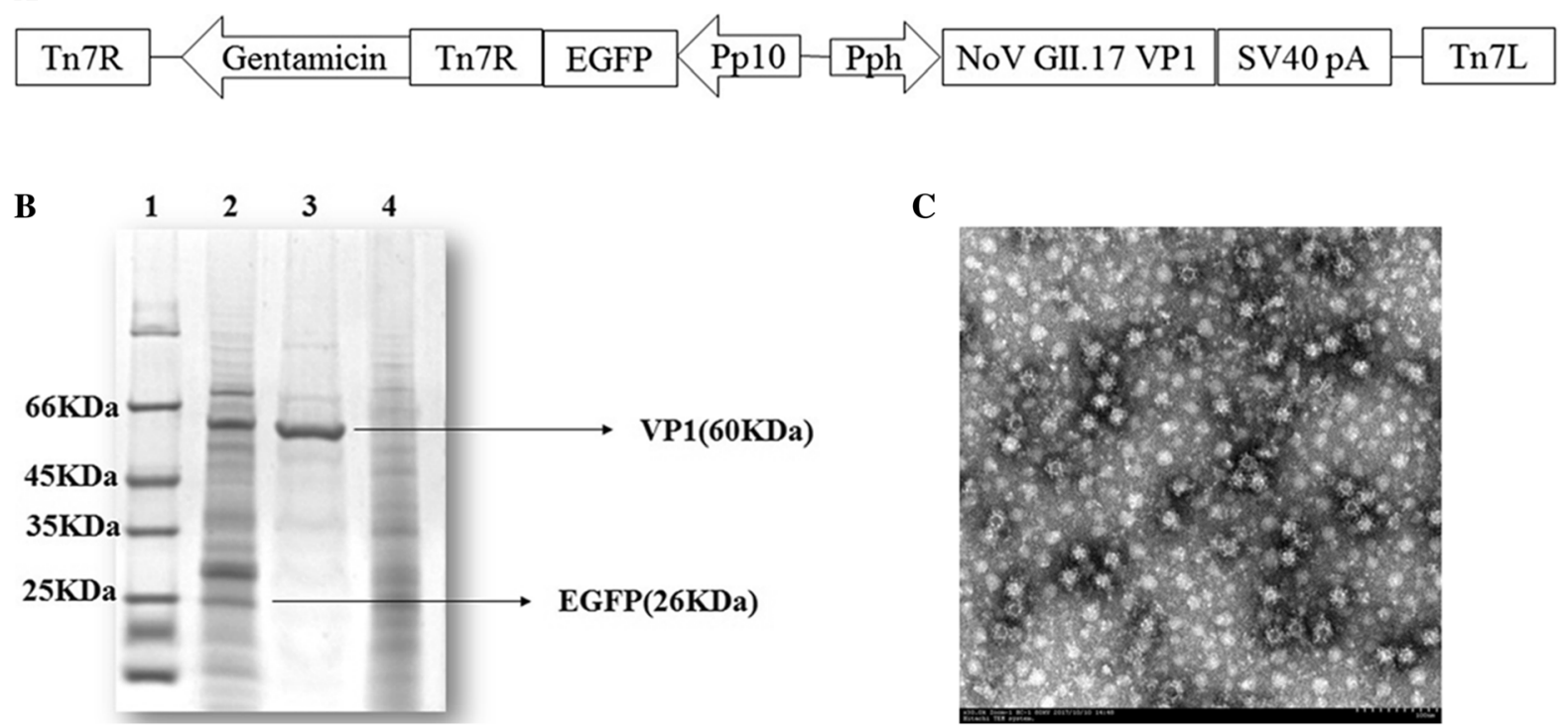

C

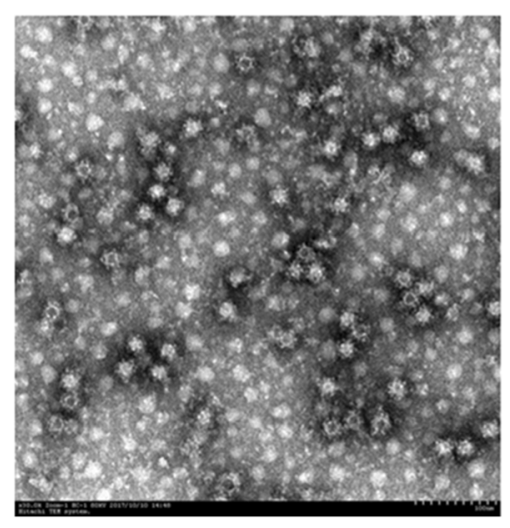

PAGE. Lane 1, Mark; Lane 2, Lysate of pFastBac-Dual-EGFPVP1 (GII.17)-infected cells; Lane 3, Purified VLPs from sucrose gradients; Lane 4, lysate of mock-infected cells. c Morphology of purified VLPs was analyzed by transmission electromicroscopy 


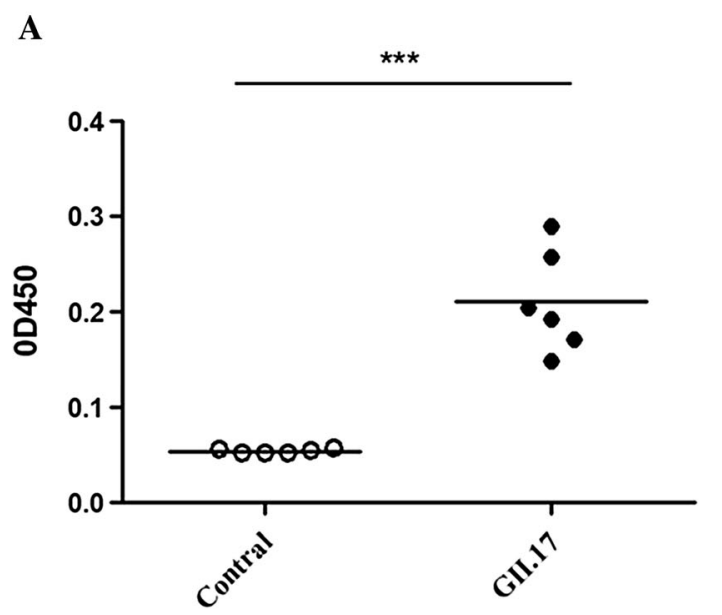

Fig. 2 Serum antibody responses in mice after immunization with GII.17 VLPs. a Serum samples were collected from each mouse 2 weeks after the final immunization and then analyzed by ELISA. Mice antisera were diluted 1:20,000. Each symbol represents an individual mouse, and the solid line indicates the

expression of VP1 protein. These results indicated that immunization with the VLPs but not the control antigen could induce GII.17 VLPs specific antibody responses in mice.

\section{Assembled VLPs bind to salivary HBGAs}

An in vitro VLP-HBGA binding assay was used to characterize the binding profiles of GII.17 capsid protein assembled VLPs. As shown in Fig. 3, VLPs bound to blood type A, B and AB salivary HBGAs, respectively, while weak binding was observed onto type $\mathrm{O}$ salivary HBGA.

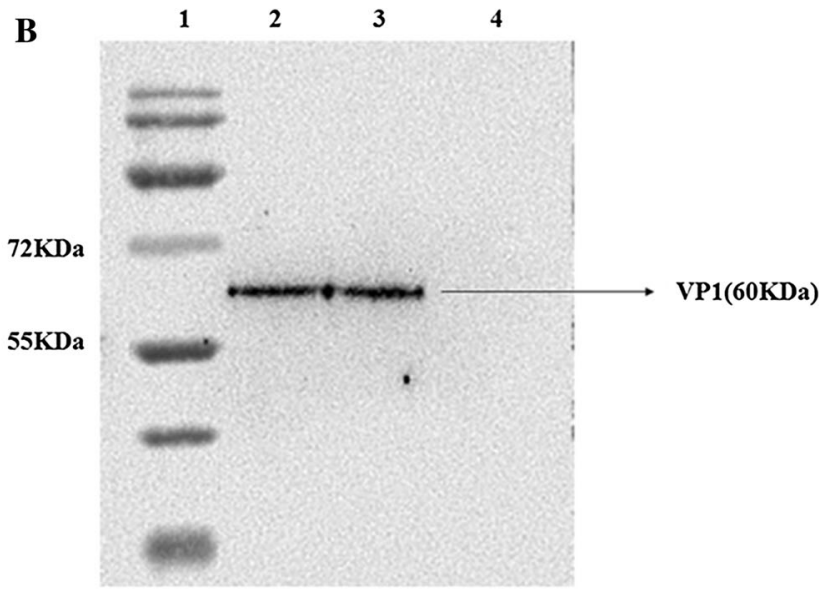

geometric mean value of the group $(* * * p<0.001)$. b Western blotting analysis of serum samples. Lane 1, Mark; Lane 2, Lysate of pFastBac-Dual-EGFP-VP1 (GII.17)-infected cells; Lane 3, Purified VLPs; Lane 4, Negative control

The capacity of VLPs immunized mouse sera to block GII.17 VLPs saliva binding

As we known, the protective function of anti-NoV serum antibodies correlates with their capacity to block NoV VLPs binding to HBGAs, therefore, the serum antibody-blocking assay was conducted to test the NoV neutralization (Atmar et al. 2011; Reeck et al. 2010; Tan and Jiang 2011). We collected the saliva HBGAs, including A, B and $\mathrm{O}$ antigens and they have therefore been used in the blockade assay. We used rabbit sera against the GII.17 P domain as antibodies in our study. Saliva samples collected from a blood type $A B$ individual was used in the in vitro VLPsHBGA binding blockade assay which represented the highest binding signal. As was shown in Fig. 4, the

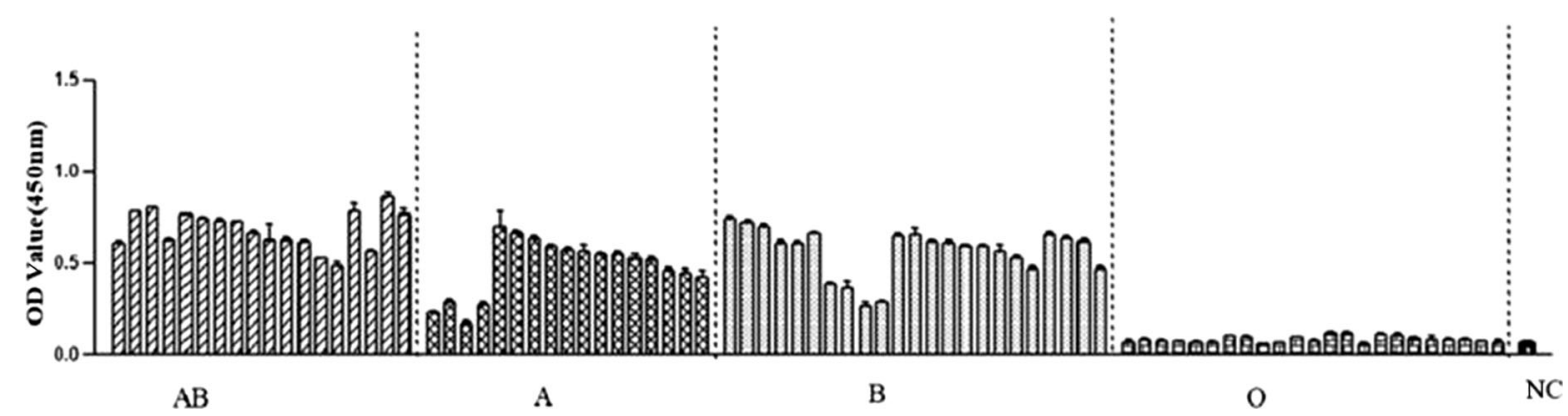

Fig. 3 Characterization of binding profiles of GII.17 VLPs to blood type A, B and AB salivary HBGAs, respectively. VLPs at 0.1 $\mu$ g/ $\mu \mathrm{L}$ were used. $N C$ negative control 


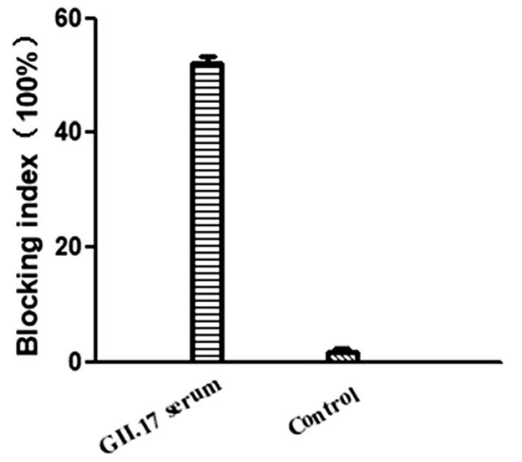

Fig. 4 VLP-HBGAs binding blockade assay. GII.17 VLPs at $0.4 \mu \mathrm{g} / \mu \mathrm{L}$ and blood type $\mathrm{AB}$ saliva were used. Rabbit antiGII.17 PP sera were diluted at 1:1000

blocking indices were higher for antisera from blood type AB. Addition of serum against GII.17 VLPs blocked the binding of GII.17-VP1 capsid protein assembled VLPs to salivary HBGAs.

\section{Discussion and conclusion}

NoVs are the major causes of acute non-bacterial gastroenteritis the worldwide, especially in children. As NoV GII.4 has rapidly evolved that produced new strains that replaced previous strains every 2 or 3 years, the recent outbreak of NoV GII.17 has sweept all over the world. Thus, it is urgent to formulate an effective and rapid NoV GII.17 vaccine. In our study, we showed that the insect cell expressed GII.17 VLPs is a promising vaccine.

The NoVs capsid protein is divided into two domains: $\mathrm{S}$ domain, forming the core of the viruslike particle, and the $\mathrm{P}$ domain, mainly involved in receptor binding (Prasad et al. 1999). By comparing the avidity of IgG from mice immunized against VLPs and $P$ particles, the results suggested that there is a significantly higher avidity for VLPs in immunized mice; moreover, it showed that immunization route and adjuvants have no effect on IgG avidity (Huo et al. 2015). Some NoV VLPs candidates have shown efficacy in human trials (Atmar et al. 2011; Bernstein et al. 2015). As we known, the VP1, which can selfassemble into VLPs when expressed in sf9 cells use a recombinant baculovirus expression system (Jiang et al. 1992). Therefore, in our research, GII.17 VLPs has been regarded as the most promising vaccine candidates. They were intraperitoneally (i.p.) injected with the antigen mixture alongside an aluminum hydroxide adjuvant.

Among the GII.17 VP1, the $\mathrm{P}$ region is further divided into the $\mathrm{P} 1$ and the $\mathrm{P} 2$ subregion. The P2 subregion is located at the outermost layer of the entire capsid, which is important for the binding of NoVs, as well as for antibody recognition (Tan et al. 2003). Regarding Escherichia coli, expression of $\mathrm{P}$ domain can lead to formation of a P-particle, 12- or 24-dimers, which contained all the elements required for receptor binding and are immunogenic in animal studies (Fang et al. 2013; Tamminen et al. 2012). So to characterize the binding specificity of the GII.17 VP1 capsid protein assembled VLPs, in our study, we used rabbit sera against the GII.17 P domain.

Due to the lack of an animal model for human NoVs infection, the challenge study could not be conducted to assess the efficacy of immunization. Recently, B cells, as a cellular target of human NoVs, could be developed in the in vitro infection model (Jones et al. 2014). Prior to that, HBGAs are important receptors for NoV infection. Also, the HBGAs-VLPs blocking assay was used as a surrogate method for the evaluation of NoVs neutralization in vitro. HBGAs have been proposed as receptors for NoVs. Due to the different genotypes of NoVs, such as GI.3 and GII.2 did not bind to synthetic or salivary HBGAs, whereas GII.4 could bind to salivary HBGAs (Huo et al. 2015, 2016a). In our study, VLPs from capsid proteins of epidemic NoV GII.17 exhibited broader HBGA recognition, except for blood type $\mathrm{O}$ individuals, however, the reason, to explain this phenomenon is unknown. A possible explanation could be sourced from a previously reported study, which found that the recombinant $\mathrm{MxV}$ (GII.3) VLPs demonstrated a degree of binding to salivary HBGAs from a blood type A individual (Huang et al. 2005).

At the same time, we used human saliva to evaluate the blockade assay. Our results indicate that rabbit serum against GII.17 VLPs, blocked the binding of capsid protein assembled VLPs to salivary HBGAs from a blood type $\mathrm{AB}$ individuals. The blocking rate reached up to $60 \%$, whereas anti-PBS did not show such effects. The discovery of new receptors or factors that promote the binding of GII.17 VLPs to HBGAs is key for monitoring the development of vaccine and vaccine efficacy evaluation. The data also suggested important information about the presence of other 
factors involved in the binding of GII.17 VLPs to HBGAs.

Considering the prevalence of GII.17, the NoVs vaccine should be urgently developed. Our results showed that GII.17 VLPs induced antibodies should be protective against in vivo NoV GII.17 infections. Therefore, we identified GII.17 VLPs as a viable candidate. NoVs vaccine represents a step forward in developing broad-spectrum, multivalent vaccines against acute viral gastroenteritis.

Acknowledgements This research was funded by the National Natural Science Foundation of China (No. 81860357).

\section{References}

Ahmed SM et al (2014) Global prevalence of norovirus in cases of gastroenteritis: a systematic review and meta-analysis. Lancet Infect Dis 14:725-730. https://doi.org/10.1016/ S1473-3099(14)70767-4

Andrade JSR, Fumian TM, Leite JPG, Assis MR, Bello G, Mir D, Miagostovich MP (2017) Detection and molecular characterization of emergent GII.P17/GII.17 Norovirus in Brazil, 2015. Infect Genet Evol 51:28-32. https://doi.org/ 10.1016/j.meegid.2017.03.011

Atmar RL et al (2011) Norovirus vaccine against experimental human Norwalk virus illness. $N$ Engl J Med 365:2178-2187. https://doi.org/10.1056/NEJMoa1101245

Bernstein DI et al (2015) Norovirus vaccine against experimental human GII.4 virus illness: a challenge study in healthy adults. J Infect Dis 211:870-878. https://doi.org/ 10.1093/infdis/jiu497

Chen $\mathrm{H}$ et al (2015) A novel norovirus GII17 lineage contributed to adult gastroenteritis in Shanghai, China, during the winter of 2014-2015. Emerg Microbes Infect 4:e67

Dai W et al (2018) A virus-like particle vaccine confers protection against enterovirus D68 lethal challenge in mice. Vaccine 36:653-659. https://doi.org/10.1016/j.vaccine. 2017.12.057

Debbink K, Donaldson EF, Lindesmith LC, Baric RS (2012) Genetic mapping of a highly variable norovirus GII.4 blockade epitope: potential role in escape from human herd immunity. J Virol 86:1214-1226. https://doi.org/10.1128/ JVI.06189-11

Fang H, Tan M, Xia M, Wang L, Jiang X (2013) Norovirus P particle efficiently elicits innate, humoral and cellular immunity. PLoS ONE 8:e63269. https://doi.org/10.1371/ journal.pone.0063269

Glass RI, Parashar UD, Estes MK (2009) Norovirus gastroenteritis. N Engl J Med 361:1776-1785. https://doi.org/10. 1056/NEJMra0804575

Huang P et al (2003) Noroviruses bind to human ABO, Lewis, and secretor histo-blood group antigens: identification of 4 distinct strain-specific patterns. J Infect Dis 188:19-31. https://doi.org/10.1086/375742
Huang XY et al (2017) A large outbreak of acute gastroenteritis caused by the human norovirus GII17 strain at a university in Henan Province. China Infect Dis Poverty 6:6. https:// doi.org/10.1186/s40249-017-0236-Z

Huang P, Farkas T, Zhong W, Tan M, Thornton S, Morrow AL, Jiang X (2005) Norovirus and histo-blood group antigens: demonstration of a wide spectrum of strain specificities and classification of two major binding groups among multiple binding patterns. J Virol 79:6714-6722

Huo Y, Wan X, Ling T, Wu J, Wang Z, Meng S, Shen S (2015) Prevailing Sydney like Norovirus GII.4 VLPs induce systemic and mucosal immune responses in mice. Mol Immunol 68:367-372. https://doi.org/10.1016/j.molimm. 2015.09.006

Huo Y, Wan X, Ling T, Shen S (2016a) Biological and immunological characterization of norovirus major capsid proteins from three different genotypes. Microb Pathog 90:78-83. https://doi.org/10.1016/j.micpath.2015.11.022

Huo Y et al (2016b) Chimeric VLPs with GII.3 P2 domain in a backbone of GII.4 VP1 confers novel HBGA binding ability. Virus Res 224:1-5. https://doi.org/10.1016/j. virusres.2016.08.006

Jiang X, Wang M, Graham DY, Estes MK (1992) Expression, self-assembly, and antigenicity of the Norwalk virus capsid protein. J Virol 66:6527-6532

Jones MK et al (2014) Enteric bacteria promote human and mouse norovirus infection of $B$ cells. Science 346:755-759. https://doi.org/10.1126/science.1257147

Kaplan JE, Feldman R, Campbell DS, Lookabaugh C, Gary GW (1982) The frequency of a Norwalk-like pattern of illness in outbreaks of acute gastroenteritis. Am J Public Health 72:1329-1332

Khamrin P et al (2016) Molecular characterization of norovirus GII.17 detected in healthy adult, intussusception patient, and acute gastroenteritis children in Thailand. Infect Genet Evol 44:330-333. https://doi.org/10.1016/j.meegid.2016. 07.031

Kroneman A et al (2013) Proposal for a unified norovirus nomenclature and genotyping. Arch Virol 158:2059-2068. https://doi.org/10.1007/s00705-013-1708-5

Lee CC, Feng Y, Chen SY, Tsai CN, Lai MW, Chiu CH (2015) Emerging Norovirus GII.17 in Taiwan. Clin Infect Dis 61:1762-1764. https://doi.org/10.1093/cid/civ647

Lin Y, Fengling L, Lianzhu W, Yuxiu Z, Yanhua J (2014) Function of VP2 protein in the stability of the secondary structure of virus-like particles of genogroup II norovirus at different $\mathrm{pH}$ levels: function of VP2 protein in the stability of NoV VLPs. J Microbiol 52:970-975. https://doi.org/10. 1007/s12275-014-4323-6

Lucero Y, Vidal R, O'Ryan GM (2017) Norovirus vaccines under development. Vaccine. https://doi.org/10.1016/j. vaccine.2017.06.043

Marionneau S, Airaud F, Bovin NV, Le Pendu J, Ruvoen-Clouet $\mathrm{N}$ (2005) Influence of the combined ABO, FUT2, and FUT3 polymorphism on susceptibility to Norwalk virus attachment. J Infect Dis 192:1071-1077. https://doi.org/10. $1086 / 432546$

Parra GI (2015) Green KY (2015) Genome of emerging Norovirus GII17, United States, 2014. Emerg Infect Dis 21:1477-1479. https://doi.org/10.3201/eid2108.150652 
Prasad BV, Hardy ME, Dokland T, Bella J, Rossmann MG, Estes MK (1999) X-ray crystallographic structure of the Norwalk virus capsid. Science 286:287-290

Ramani S, Atmar RL, Estes MK (2014) Epidemiology of human noroviruses and updates on vaccine development. Curr Opin Gastroenterol 30:25-33. https://doi.org/10.1097/ MOG.0000000000000022

Reeck A, Kavanagh O, Estes MK, Opekun AR, Gilger MA, Graham DY, Atmar RL (2010) Serological correlate of protection against norovirus-induced gastroenteritis. J Infect Dis 202:1212-1218. https://doi.org/10.1086/656364

Tamminen K, Huhti L, Koho T, Lappalainen S, Hytonen VP, Vesikari T, Blazevic V (2012) A comparison of immunogenicity of norovirus GII-4 virus-like particles and P-particles. Immunology 135:89-99. https://doi.org/10.1111/j. 1365-2567.2011.03516.x

Tan M, Jiang X (2005) The p domain of norovirus capsid protein forms a subviral particle that binds to histo-blood group antigen receptors. J Virol 79:14017-14030. https://doi.org/ 10.1128/JVI.79.22.14017-14030.2005

Tan M, Jiang X (2011) Norovirus-host interaction: multi-selections by human histo-blood group antigens. Trends Microbiol 19:382-388. https://doi.org/10.1016/j.tim.2011. 05.007
Tan M, Huang P, Meller J, Zhong W, Farkas T, Jiang X (2003) Mutations within the $\mathrm{P} 2$ domain of norovirus capsid affect binding to human histo-blood group antigens: evidence for a binding pocket. J Virol 77:12562

Zhang C et al (2015) High-yield production of recombinant virus-like particles of enterovirus 71 in Pichia pastoris and their protective efficacy against oral viral challenge in mice. Vaccine 33:2335-2341. https://doi.org/10.1016/j. vaccine.2015.03.034

Zhang C, Liu Q, Ku Z, Hu Y, Ye X, Zhang Y, Huang Z (2016) Coxsackievirus A16-like particles produced in Pichia pastoris elicit high-titer neutralizing antibodies and confer protection against lethal viral challenge in mice. Antiviral Res 129:47-51. https://doi.org/10.1016/j.antiviral.2016. 02.011

Zheng DP, Ando T, Fankhauser RL, Beard RS, Glass RI, Monroe SS (2006) Norovirus classification and proposed strain nomenclature. Virology 346:312-323. https://doi. org/10.1016/j.virol.2005.11.015

Publisher's Note Springer Nature remains neutral with regard to jurisdictional claims in published maps and institutional affiliations. 